\title{
AUTOEFICÁCIA DISCENTE NA FORMAÇÃO INICIAL DE ESTUDANTES UNIVERSITÁRIOS DE EDUCAÇÃO FÍSICA
}

\author{
STUDENTS' SELF-EFFICACY IN INITIAL UNIVERSITY TRAINING IN \\ PHYSICAL EDUCATION
}

\section{AUTOEFICACIA DISCENTE EN LA FORMACION INICIAL DE ESTUDIANTES UNIVERSITARIOS DE EDUCACIÓN FÍSICA}

\author{
William das Neves Salles*, Juarez Vieira do Nascimento*, \\ Júlio César Schmitt Rocha*, Edison Roberto de Souza*
}

\section{Palavras chave: \\ Eficácia. \\ Estudantes. \\ Universidades. \\ Competência \\ profissional.}

Keywords:

Efficacy.

Students.

Universities.

Professional

Competence.

Palabras clave:

Eficacia.

Estudiantes.

Universidades.

Competencia

profesional.

\begin{abstract}
Resumo: Analisaram-se as relações da autoeficácia (AE) discente com aspectos da formação inicial de 385 estudantes universitários de Educação Física. Foram aplicadas a Escala de Autoeficácia na Formação Superior e a Escala de Motivação Acadêmica aos estudantes, e os resultados revelaram diferenças significativas na AE discente, considerando-se as variáveis sexo, curso realizado, participação em atividades de pesquisa e extensão, Índice de Aproveitamento Acumulado e Índice de Autodeterminação. As evidências ressaltam a importância da investigação aprofundada da $A E$ no ensino superior, que pode contribuir para o desenvolvimento acadêmicoprofissional do estudante e para a melhoria da qualidade dos cursos de graduação ofertados.
\end{abstract}

Abstract: Students' self-efficacy relations were analyzed, together with aspects of initial training of 385 Physical Education university students. The Higher Education Self-Efficacy Scale and the Academic Motivation Scale were applied to students, and results showed statistically significant differences between students' self-efficacy, considering the variables of gender, major, participation in research and extension activities, Academic Achievement Index, and Self-Determination Index. Evidence shows the importance of thorough investigation of SE in Higher Education, which may contribute to students' academic and professional development and to improving the quality of undergraduate courses offered.

Resumen: Se analizaron las relaciones de la autoeficacia (AE) discente con aspectos de la formación inicial de 385 estudiantes universitarios de Educación Física. Se aplicaron la Escala de Autoeficacia en la Educación Superior y la Escala de Motivación Académica a los estudiantes, y los resultados revelaron diferencias significativas en la AE discente considerando sexo, curso, participación en actividades de investigación y extensión, Índice de Rendimiento Académico e Índice de Autodeterminación. Las evidencias demuestran la importancia de investigar la AE discente en la Educación Superior, que puede contribuir para el desarrollo académico profesional del estudiante y para mejorar la calidad de los cursos de graduación ofrecidos.
*Universidade Federal de Santa Catarina. Florianópolis, SC, Brasil. E-mail: williamdnsalles@gmail.com

Recebido em: 14-03-2015 Aprovado em: 18-08-2015 (c) (1) (8) Licence 


\section{INTRODUÇÃO}

O período de formação inicial universitária é muito rico e desafiador ao estudante, em decorrência da complexidade, da dinamicidade e das peculiaridades inerentes ao ambiente acadêmico, bem como dos atributos pessoais dinâmicos de cada indivíduo.

Um dos principais objetivos da formação inicial é promover o desenvolvimento de competências profissionais nos estudantes. Oprocesso de desenvolvimento dessas competências, por sua vez, é influenciado pelo perfil de egresso almejado pela instituição de ensino superior, o que acaba se refletindo na eleição dos conteúdos programáticos das disciplinas, nas abordagens metodológicas de ensino e de avaliação e na criação de tempos e espaços específicos de vivência para os estudantes em formação (VIEIRA; COIMBRA 2006).

O aproveitamento do curso pelo estudante e o consequente desenvolvimento de competências acadêmico-profissionais, entretanto, não são influenciados somente pelas características objetivas do ambiente universitário, mas, também, pelas percepções subjetivas acerca deste ambiente e de si mesmo, motivo pelo qual os julgamentos pessoais sobre esses aspectos assumem importante papel ao longo da trajetória profissional (MASCARENHAS, 2010).

Dentre os aspectos que podem ser objeto de autorreflexão, merece destaque a autoeficácia $(A E)$, caracterizada como sendo o conjunto de crenças pessoais sobre a própria capacidade de executar ações para atingir determinadas realizações (BANDURA, 1977). As percepções de $A E$ exercem importante influência sobre a maneira que o indivíduo se relaciona com o ambiente em que está inserido, pois podem determinar as opções e as atitudes individuais, bem como influenciar a quantidade de energia, tempo e perseverança a ser despendida em prol de um objetivo determinado (BANDURA, 1977; 2008). Além disso, a percepção de AE resulta de complexas interações que ocorrem entre as características pessoais e comportamentais dos indivíduos com fatores externos presentes nos distintos ambientes em que se fazem presentes, de maneira que as explicações sobre suas ações podem ser também encontradas nas inter-relações entre as especificidades pessoais e os ambientes passados e presentes (BANDURA, 1997).

No âmbito acadêmico, o estudo e a avaliação das crenças de AE são importantes pois possibilitam ampliar o conhecimento das características envolvidas no processo de ensinoaprendizagem (SILVA; IAOCHITE; AZZI, 2010), bem como coordenar ações de orientação educativa que fortaleçam os comportamentos preditivos de eficiência e de qualidade da aprendizagem (MASCARENHAS, 2010). A AE discente compreende um conjunto de crenças de um estudante em sua capacidade de organizar e executar cursos de ações requeridos para produzir determinadas realizações no âmbito do ensino superior. De acordo com Polydoro e Guerreiro-Casanova (2010), a AE discente apresenta cinco dimensões: AE Acadêmica; AE na Regulação da Formação; AE na Interação Social; AE em Ações Proativas; AE na Gestão Acadêmica, cuja avaliação contribui para melhor compreender o comportamento do estudante frente ao respectivo curso de formação inicial realizado.

Investigações têm demonstrado que as crenças de AE discente influenciam o modo como um estudante sente, pensa, motiva-se e comporta-se, o que faz com que desempenhem papel basilar sobre o agir individual (POLYDORO; GUERREIRO-CASANOVA, 2010). Com efeito, a percepção de AE discente exerce influência sobre escolhas (FOLTZ; LUZZO, 1998), estabelecimento de metas (ELIAS; LOOMIS, 2002), esforço despendido com tarefas universitárias (KOMARRAJU; NADLER, 2013), perseverança frente às adversidades (LI; NISHIKAWA, 2012), 
crenças de sucesso (LOPES; TEIXEIRA, 2012) e, por fim, desempenho acadêmico do estudante (AL KHATIB, 2010).

Apesar da realização de estudos internacionais e nacionais sobre AE discente no âmbito da formação inicial universitária, ainda se observa importante carência investigativa na área da Educação Física, em especial após as mais recentes mudanças curriculares que resultaram na oferta de cursos distintos de bacharelado e de licenciatura. Neste sentido, optou-se pela investigação da seguinte questão: quais são as relações da $\mathrm{AE}$ discente com aspectos da formação inicial de estudantes universitários de Educação Física?

\section{PROCEDIMENTOS METODOLÓGICOS}

Este estudo descritivo-correlacional e empírico classifica-se como aplicado quanto à sua natureza, utilizando-se da abordagem quantitativa para investigação (THOMAS; NELSON; SILVERMAN, 2007). A população-alvo foi composta pelos 490 estudantes de Educação Física da Universidade Federal de Santa Catarina (UFSC) cadastrados junto à coordenadoria dos respectivos cursos no primeiro semestre de 2014.

A formação inicial em Educação Física, na instituição investigada, é oferecida desde 1974. A partir de 2006, em decorrência das Resoluções CNE no 01 (BRASIL, 2002a), CNE no 02 (BRASIL, 2002b) e CNE no 07 (BRASIL, 2004), passou-se a oferecer duas habilitações (licenciatura e bacharelado) para o exercício profissional em Educação Física, com entradas distintas e prazos iguais de integralização curricular (mínimo de oito e máximo de 14 semestres). Enquanto que o curso de licenciatura (3.480 horas/aula) está estruturado para formar professores para intervir no componente curricular Educação Física da Educação Básica e Profissional, o curso de bacharelado (3.840 horas/aula) está direcionado à formação de profissionais para intervir nos âmbitos da avaliação e prescrição de exercícios, atividade física na promoção da saúde e gestão e treinamento esportivo.

Não foram convidados a participar da pesquisa os estudantes intercambistas provenientes de outras instituições, os estudantes com idade inferior a 18 anos e os estudantes que não compareceram regularmente às aulas após a metade do semestre letivo. Considerando os critérios estabelecidos, 412 estudantes ficaram elegíveis para a participação na investigação. Destes, 385 consentiram em participar e compuseram a amostra do presente estudo.

Após a autorização da instituição investigada e a aprovação do projeto pelo Comitê de Ética em Pesquisa com Seres Humanos (Parecer n 507.318/2014), foram solicitadas informações à coordenadoria dos cursos sobre as cargas horárias curriculares cumpridas por cada estudante até 0 semestre anterior à coleta de dados, a fim de que pudesse ser estabelecido o nível de progressão individual desses indivíduos na formação inicial.

Na sequência desse processo foi realizado o contato direto (em sala de aula) com os estudantes presentes para explicar a natureza e os objetivos do estudo, bem como para destacar o caráter voluntário da participação e obter os devidos consentimentos para a participação. Aos estudantes que aceitaram participar, foram aplicados a Escala de Autoeficácia na Formação Superior (EAFS) (POLYDORO; GUERREIRO-CASANOVA, 2010), a Escala de Motivação Acadêmica (EMA) (SOBRAL, 2003) e um questionário sociodemográfico, os quais foram respondidos em sala de aula. Aos estudantes que não se encontravam em sala no momento da coleta, foram encaminhadas via e-mail as respectivas versões online dos instrumentos, as quais foram criadas a partir da plataforma Google Docs. 
A EAFS busca identificar as percepções de AE discente, sendo composta por 34 itens que se distribuem em cinco dimensões: AE Acadêmica (capacidade de aprender e aplicar conhecimento); AE na Regulação da Formação (capacidade de autorregular as ações); AE na Interação Social (capacidade de relacionamento com colegas e professores); AE em Ações Proativas (capacidade de aproveitar e promover oportunidades de formação); AE na Gestão Acadêmica (capacidade de envolver-se e cumprir prazos). O respondente deve assinalar, em cada um dos itens e considerando uma escala de 0 (pior percepção) a 10 (melhor percepção), 0 conceito que mais se adequa à sua percepção de $\mathrm{AE}$.

A EMA (VALLERAND et al., 1993) foi traduzida para a língua portuguesa e aplicada no Brasil pela primeira vez por Sobral (2003), junto aos universitários de um curso de Medicina. Este instrumento constitui-se de 28 itens, distribuídos em sete dimensões: Desmotivação (ausência de percepção de contingências entre as ações e seus desfechos/falta de motivos intrínsecos ou extrínsecos); Regulação externa (fazer algo porque se sente pressionado por outros a fazêlo); Regulação introjetada (fazer algo porque se pressiona a si próprio a fazê-lo); Regulação identificada (fazer algo porque se decidiu fazê-lo); Motivação intrínseca para vivenciar estímulo (fazer algo a fim de experimentar sensações estimulantes, de natureza sensorial ou estética); Motivação intrínseca para realizar coisas (fazer algo pelo prazer e satisfação que decorrem da busca de realização ou criação de coisas); Motivação intrínseca para saber (fazer algo pelo prazer e satisfação que decorrem de aprender, explorar ou entender) (SOBRAL, 2008). As respostas da EMA são assinaladas de acordo com o grau de correspondência que o respondente identifica em cada item com sua percepção pessoal, levando-se em consideração uma escala que varia de 1 (nenhuma correspondência) a 7 (total correspondência).

As informações coletadas foram analisadas com auxílio do software SPSS Statistics (versão 21.0), por intermédio da utilização de recursos estatísticos descritivos (frequência absoluta e percentual; média e desvio-padrão) e inferenciais (testes de hipóteses). Para a análise do nível de associação entre as percepções de AE discente e as variáveis pessoais (sexo; estado civil; faixa etária; Índice de Autodeterminação), acadêmicas (curso; participação em atividades de pesquisa; participação em atividades de extensão; nível de progressão no curso; Índice de Aproveitamento Acumulado) e profissionais (vínculo empregatício) foi aplicado o teste Quiquadrado, considerando-se o nível de significância de $5 \%$.

O Índice de Aproveitamento Acumulado (IAA) é calculado cumulativamente a cada semestre, sendo representado pelo quociente entre o somatório de pontos obtidos (ou seja, as notas obtidas pelos estudantes multiplicadas pela carga horária de cada disciplina cursada) e a carga horária matriculada (UNIVERSIDADE FEDERAL DE SANTA CATARINA, 1997).

A alteração na categorização original da variável AE (POLYDORO; GUERREIROCASANOVA, 2010) foi necessária diante das baixas incidências de valores encontradas, no presente estudo, nas categorias "baixa" e "alta". Assim, optou-se pelo agrupamento das categorias "baixa e muito baixa" " "alta e muito alta" para facilitar a aplicação do teste Qui-quadrado (conforme indicado por MARÔCO, 2010, p. 104). As categorias adotadas neste estudo foram: "muito baixa ou baixa" (valores até 6,9) "média" (de 7 a 8,9) e "alta ou muito alta" (9 ou mais).

A variável Índice de Autodeterminação (Self-Determination Index, ou SDI), por sua vez, integra em medida única as diferentes dimensões da motivação avaliadas pela EMA. No presente estudo, o SDI foi calculado por meio da seguinte fórmula: [( $3^{\star}(\mathrm{Ml}$ para saber $)+2^{\star}(\mathrm{MI}$ para realizar coisas $)+1^{*}(\mathrm{Ml}$ para vivenciar estímulo + regulação identificada $)-\left(1^{*}\right.$ (regulação 
externa $)+2^{*}$ (regulação introjetada $)+3^{\star}$ (desmotivação) $)$. O SDI resultante, que pode oscilar em um continuum de $-48 \mathrm{a}+48$, foi posteriormente categorizado da seguinte maneira: negativo (de -48 até 0), que corresponde à predominância de motivação extrínseca no indivíduo; positivo (de 1 a 48), correspondendo à predominância de motivação intrínseca no indivíduo. Os procedimentos descritos para atribuição de ponderações e cálculo do SDI encontram divergências na literatura consultada. Desta maneira, optou-se por realizar pequena adaptação na fórmula original descrita em Guay, Megeau e Vallerand (2003), considerando a presença do autor da escala original nesta proposta de cálculo.

\section{RESULTADOS}

A Tabela 1 apresenta informações de caracterização dos 385 estudantes universitários investigados. De maneira geral, constatou-se predominância de indivíduos do sexo masculino, com faixa etária de 20 a 24 anos, solteiros e com vínculo empregatício. A maior parte dos estudantes cursava licenciatura e não tinha participado de atividades acadêmicas de extensão ou de pesquisa até a coleta de dados, possuindo IAA predominantemente regular ou bom e SDI negativo. A maior proporção dos universitários investigados se encontrava na primeira etapa da formação, ao passo que o menor contingente estava próximo da conclusão do curso.

Tabela 1 - Características pessoais, acadêmicas e profissionais dos estudantes investigados

\begin{tabular}{|c|c|c|}
\hline Variáveis & Categorias & $\mathrm{n}(\%)$ \\
\hline \multirow{2}{*}{ Sexo } & Masculino & $223(57,9)$ \\
\hline & Feminino & $162(42,1)$ \\
\hline \multirow{4}{*}{ Faixa etária } & Até 20 anos & $47(12,2)$ \\
\hline & 20 a 24 anos & $199(51,7)$ \\
\hline & 25 a 29 anos & $95(24,7)$ \\
\hline & 30 anos ou mais & $44(11,4)$ \\
\hline \multirow{2}{*}{ Estado civil } & Solteiro(a) & $363(94,3)$ \\
\hline & Outros & $22(5,7)$ \\
\hline \multirow{2}{*}{ Vínculo empregatício } & Não & $104(27,0)$ \\
\hline & Sim & $281(73,0)$ \\
\hline \multirow{2}{*}{ Curso } & Bacharelado & $183(47,5)$ \\
\hline & Licenciatura & $202(52,5)$ \\
\hline \multirow{2}{*}{ Participação em pesquisa } & Não & $291(75,6)$ \\
\hline & Sim & $94(24,4)$ \\
\hline \multirow{2}{*}{ Participação em extensão } & Não & $198(51,4)$ \\
\hline & Sim & $187(48,6)$ \\
\hline \multirow{2}{*}{ Índice de Aproveitamento Acumulado } & Regular ou bom (Até 8,5) & $343(89,1)$ \\
\hline & Muito bom ou ótimo $(>8,5)$ & $42(10,9)$ \\
\hline \multirow{4}{*}{ Nível de progressão no curso } & Até $25 \%$ & $120(31,2)$ \\
\hline & 25,01 a $50 \%$ & $91(23,6)$ \\
\hline & 50,01 a $75 \%$ & $99(25,7)$ \\
\hline & Mais de $75 \%$ & $75(19,5)$ \\
\hline \multirow{2}{*}{ Índice de Autodeterminação } & Negativo & $209(54,3)$ \\
\hline & Positivo & $176(45,7)$ \\
\hline
\end{tabular}


A análise descritiva dos níveis de AE discente geral e específica por dimensão (Tabela 2) possibilitou identificar que os indivíduos do sexo feminino, com até 24 anos, casados/ divorciados e com vínculo empregatício (seja na área de Educação Física ou em outras áreas) apresentaram escores superiores, tanto no âmbito geral quanto nas dimensões específicas. Quando consideradas as características acadêmicas, observou-se que os estudantes do curso de bacharelado, com melhores índices de aproveitamento acadêmico e de autodeterminação, bem como com experiências prévias em atividades de extensão e pesquisa, apresentaram os maiores níveis de $\mathrm{AE}$ discente.

Tabela 2 - Níveis de AE geral e específica dos estudantes, considerando variáveis pessoais, acadêmicas e profissionais.

\begin{tabular}{|c|c|c|c|c|c|c|}
\hline \multirow[b]{2}{*}{ Variáveis e categorias } & \multicolumn{6}{|c|}{ AE discente } \\
\hline & Acadêmica & Regulação & $\begin{array}{c}\text { Ações } \\
\text { proativas }\end{array}$ & $\begin{array}{l}\text { Interação } \\
\text { social }\end{array}$ & $\begin{array}{c}\text { Gestão } \\
\text { acadêmica }\end{array}$ & Geral \\
\hline \multicolumn{7}{|l|}{ Sexo } \\
\hline Masculino & $7,72(1,29)$ & $7,60(1,57)$ & $6,85(1,74)$ & $8,23(1,26)$ & $7,58(1,66)$ & $7,60(1,31)$ \\
\hline Feminino & $8,21(1,21)$ & $8,07(1,38)$ & $7,50(1,48)$ & $8,68(1,16)$ & $8,43(1,31)$ & $8,16(1,15)$ \\
\hline \multicolumn{7}{|l|}{ Faixa etária } \\
\hline Até 24 anos & $7,96(1,12)$ & $7,78(1,38)$ & $7,09(1,60)$ & $8,50(1,09)$ & $8,01(1,50)$ & $7,86(1,13)$ \\
\hline 25 anos ou mais & $7,86(1,53)$ & $7,82(1,71)$ & $7,17(1,79)$ & $8,28(1,45)$ & $7,82(1,70)$ & $7,79(1,50)$ \\
\hline \multicolumn{7}{|l|}{ Estado civil } \\
\hline Solteiro(a) & $7,92(1,23)$ & $7,78(1,49)$ & $7,11(1,66)$ & $8,43(1,18)$ & $7,93(1,54)$ & $7,83(1,24)$ \\
\hline Outros & $7,93(1,98)$ & $8,06(1,73)$ & $7,41(1,89)$ & $8,27(2,01)$ & $8,09(2,10)$ & $7,94(1,84)$ \\
\hline \multicolumn{7}{|l|}{ Vínculo empregatício } \\
\hline Não & $7,85(1,12)$ & $7,48(1,54)$ & $6,99(1,63)$ & $8,32(1,15)$ & $7,79(1,47)$ & $7,69(1,18)$ \\
\hline Sim & $7,95(1,34)$ & $7,91(1,48)$ & $7,17(1,68)$ & $8,46(1,27)$ & $8,00(1,61)$ & $7,89(1,31)$ \\
\hline \multicolumn{7}{|l|}{ Curso } \\
\hline Bacharelado & $8,17(1,20)$ & $8,05(1,47)$ & $7,26(1,70)$ & $8,48(1,18)$ & $8,08(1,62)$ & $8,01(1,23)$ \\
\hline Licenciatura & $7,71(1,32)$ & $7,57(1,51)$ & $7,00(1,64)$ & $8,36(1,29)$ & $7,81(1,52)$ & $7,67(1,30)$ \\
\hline \multicolumn{7}{|l|}{ Participação em pesquisa } \\
\hline Não & $7,77(1,25)$ & $7,68(1,52)$ & $6,91(1,68)$ & $8,30(1,23)$ & $7,72(1,62)$ & $7,68(1,27)$ \\
\hline Sim & $8,39(1,27)$ & $8,14(1,42)$ & $7,78(1,46)$ & $8,78(1,20)$ & $8,61(1,22)$ & $8,32(1,18)$ \\
\hline \multicolumn{7}{|l|}{ Participação em extensão } \\
\hline Não & $7,79(1,30)$ & $7,68(1,56)$ & $6,82(1,78)$ & $8,25(1,26)$ & $7,77(1,68)$ & $7,65(1,33)$ \\
\hline Sim & $8,07(1,24)$ & $7,92(1,45)$ & $7,44(7,57)$ & $8,60(1,18)$ & $8,12(1,44)$ & $8,03(1,20)$ \\
\hline \multicolumn{7}{|c|}{ Índice de Aproveitamento Acumulado } \\
\hline Regular ou bom (Até 8,5) & $7,82(1,28)$ & $7,73(1,53)$ & $7,04(1,67)$ & $8,34(1,24)$ & $7,79(1,57)$ & $7,75(1,27)$ \\
\hline Muito bom ou ótimo $(>8,5)$ & $8,78(0,97)$ & $8,29(1,24)$ & $7,78(1,49)$ & $9,05(1,02)$ & $9,14(1,01)$ & $8,55(1,11)$ \\
\hline \multicolumn{7}{|l|}{ Nível de progressão no curso } \\
\hline Até $25 \%$ & $7,86(1,33)$ & $7,69(1,63)$ & $6,98(1,80)$ & $8,36(1,35)$ & $7,98(1,65)$ & $7,75(1,38)$ \\
\hline 25,01 a $50 \%$ & $7,91(1,19)$ & $7,82(1,42)$ & $7,14(1,64)$ & $8,49(1,12)$ & $8,13(1,51)$ & $7,88(1,20)$ \\
\hline 50,01 a $75 \%$ & $7,87(1,23)$ & $7,70(1,52)$ & $7,06(1,56)$ & $8,32(1,07)$ & $7,70(1,50)$ & $7,74(1,20)$ \\
\hline Mais de $75 \%$ & $8,12(1,37)$ & $8,05(1,39)$ & $7,41(1,62)$ & $8,57(1,38)$ & $7,97(1,62)$ & $8,04(1,30)$ \\
\hline \multicolumn{7}{|l|}{ Índice de Autodeterminação } \\
\hline Negativo & $7,54(1,37)$ & $7,35(1,67)$ & $6,54(1,77)$ & $8,07(1,39)$ & $7,39(1,64)$ & $7,38(1,37)$ \\
\hline Positivo & $8,38(0,99)$ & $8,33(1,07)$ & $7,82(1,23)$ & $8,83(0,87)$ & $8,60(1,20)$ & $8,37(0,90)$ \\
\hline
\end{tabular}


Com a finalidade de identificar o nível de associação entre a $A E$ discente e variáveis pessoais, acadêmicas e profissionais (Tabela 3), a AE foi analisada considerando a proposta de categorização mencionada nos procedimentos metodológicos. A análise inferencial confirmou a existência de associações estatisticamente significativas da AE com o sexo (feminino>masculino), o curso (bacharelado>licenciatura), a participação em atividades de pesquisa (sim>não) e extensão (sim>não), o IAA (ótimo>regular) e o SDI (positivo>negativo) dos estudantes investigados.

Tabela 3 - Nível de associação entre a AE geral dos estudantes e variáveis pessoais, acadêmicas e profissionais

\begin{tabular}{|c|c|c|c|c|}
\hline \multirow[b]{2}{*}{ Variáveis e categorias } & \multicolumn{3}{|c|}{ AE discente } & \multirow[b]{2}{*}{$\mathrm{p}$-valor } \\
\hline & $\begin{array}{l}\text { Muito baixa ou } \\
\text { Baixa (\%) }\end{array}$ & Média (\%) & $\begin{array}{l}\text { Alta ou Muito alta } \\
(\%)\end{array}$ & \\
\hline \multicolumn{5}{|l|}{ Sexo } \\
\hline Masculino & $49(22,0)$ & $145(65,0)$ & $29(13,0)$ & \multirow{2}{*}{$<0,001$} \\
\hline Feminino & $16(9,9)$ & $96(59,3)$ & $50(30,9)$ & \\
\hline \multicolumn{5}{|l|}{ Faixa etária } \\
\hline Até 24 anos & $38(15,4)$ & $158(64,2)$ & $50(20,3)$ & \multirow{2}{*}{0,57} \\
\hline 25 anos ou mais & $27(19,4)$ & $83(59,7)$ & $29(20,9)$ & \\
\hline \multicolumn{5}{|l|}{ Estado civil } \\
\hline Solteiro(a) & $62(17,1)$ & $228(62,8)$ & $73(20,1)$ & \multirow{2}{*}{0,70} \\
\hline Outros & $3(13,6)$ & $13(59,1)$ & $6(27,3)$ & \\
\hline \multicolumn{5}{|l|}{ Vínculo empregatício } \\
\hline Não & $20(19,2)$ & $69(66,3)$ & $15(14,4)$ & \multirow{2}{*}{0,18} \\
\hline Sim & $45(16,0)$ & $172(61,2)$ & $64(22,8)$ & \\
\hline \multicolumn{5}{|l|}{ Curso } \\
\hline Bacharelado & $30(16,4)$ & $101(55,2)$ & $52(28,4)$ & \multirow{2}{*}{$<0,001$} \\
\hline Licenciatura & $35(17,3)$ & $140(69,3)$ & $27(13,4)$ & \\
\hline \multicolumn{5}{|l|}{ Participação em pesquisa } \\
\hline Não & $57(19,6)$ & $188(64,6)$ & $46(15,8)$ & \multirow{2}{*}{$<0,001$} \\
\hline Sim & $8(8,5)$ & $53(56,4)$ & $33(35,1)$ & \\
\hline \multicolumn{5}{|l|}{ Participação em extensão } \\
\hline Não & $46(23,2)$ & $110(55,6)$ & $42(21,2)$ & \multirow{2}{*}{$<0,001$} \\
\hline Sim & $19(10,2)$ & $131(70,1)$ & $37(19,8)$ & \\
\hline \multicolumn{5}{|l|}{ Índice de Aproveitamento Acumulado } \\
\hline Regular ou bom (Até 8,5 ) & $64(18,7)$ & $218(63,6)$ & $61(17,8)$ & \multirow{2}{*}{$<0,001$} \\
\hline Muito bom ou ótimo (Mais de 8,5 ) & $1(2,4)$ & $23(54,8)$ & $18(42,9)$ & \\
\hline \multicolumn{5}{|l|}{ Nível de progressão no curso } \\
\hline Até $25 \%$ & $23(19,2)$ & $72(60,0)$ & $25(20,8)$ & \multirow{4}{*}{0,64} \\
\hline 25,01 a $50 \%$ & $13(14,3)$ & $58(63,7)$ & $20(22,0)$ & \\
\hline 50,01 a $75 \%$ & $19(19,2)$ & $65(65,7)$ & $15(15,1)$ & \\
\hline Mais de $75 \%$ & $10(13,3)$ & $46(61,4)$ & $19(25,3)$ & \\
\hline \multicolumn{5}{|l|}{ Índice de Autodeterminação } \\
\hline Negativo & $54(25,8)$ & $133(63,6)$ & $22(10,5)$ & \multirow{2}{*}{$<0,001$} \\
\hline Positivo & $11(6,3)$ & $108(61,4)$ & $57(32,4)$ & \\
\hline
\end{tabular}




\section{DISCUSSÃO}

Preliminarmente à discussão dos resultados, é importante destacar a presença de limitações no presente estudo. Neste sentido, salienta-se que não foi realizado o acompanhamento efetivo (observação sistemática) do comportamento discente dentro e fora da universidade, nem do nível de autoexigência individual, os quais poderiam auxiliar na compreensão aprofundada tanto das relações pedagógicas que se estabelecem entre os membros do corpo social do curso como da conduta acadêmica dos estudantes investigados. Além disso, e considerando que a coleta de dados ocorreu após a metade do semestre letivo, não foram incluídos na amostra os estudantes desistentes dos cursos investigados, cujas percepções de AE poderiam revelar aspectos relevantes acerca da formação inicial oferecida pela instituição.

As evidências encontradas nesta investigação indicaram que as estudantes do sexo feminino apresentaram maiores níveis de $\mathrm{AE}$ do que os indivíduos do sexo masculino. $\mathrm{Na}$ literatura consultada, não parece existir consenso nas comparações dos níveis de $A E$ discente entre os sexos. Enquanto que parte das investigações não tem encontrado associação (VELIZBURGOS; URQUIJO, 2012, CELIK, 2013), há estudos que indicam maiores níveis de AE discente em estudantes do sexo masculino (CENDALES; VARGAS-TRUJILLO; BARBOSA, 2013, SÁNCHEZ ROSAS, 2013) e outros que encontraram associação com o sexo feminino (OZAN et al., 2012, VEGA et al., 2012). Entretanto, a literatura consultada apresenta investigações que indicam que as mulheres apresentam maiores níveis de autorregulação (OZAN et al., 2012), atenção à fala dos professores em sala de aula e planejamento para tarefas e exames (VEGA et al., 2012) do que os homens. A maior habilidade autorregulatória, bem como o maior nível de atenção despendido aos professores, pode aumentar a percepção de controle das mulheres em relação a ambientes complexos como o da formação inicial, contribuindo para melhor ajustamento ao curso (CAZAN, 2012) e, consequentemente, para o aumento das chances de realização acadêmica e de obtenção de desempenhos mais elevados.

Outro importante aspecto a destacar é a maneira diferente que estudantes de ambos os sexos processam as informações provindas das quatro fontes de $\mathrm{AE}$ (experiências diretas; experiências vicárias; estados fisiológicos e emocionais; persuasão verbal/social), o que tem implicações diretas sobre o grau de significância de determinado acontecimento ou experiência sobre o comportamento acadêmico posterior (VEGA et al., 2012). Em investigação realizada com 352 estudantes universitários de Física de uma universidade nos EUA, Sawtelle, Brewe e Kramer (2012) examinaram a AE discente sob a perspectiva da teoria do gênero, no intuito de compreender como as fontes de AE provêm mecanismos para entender o processo de retenção/reprovação em Física. Os resultados indicaram que a $A E$ revelou-se boa preditora do sucesso acadêmico nos estudantes investigados e, considerando especificamente as mulheres, que a predição da probabilidade de aprovação delas em Física dependeu, primeiramente, das experiências vicárias, sem contribuição significante da persuasão verbal/social. Desta maneira, além dos maiores níveis de autorregulação na formação, as estudantes investigadas no presente estudo podem se beneficiar mais das experiências vicárias para a construção de sua $\mathrm{AE}$ discente, considerando que observam e prestam mais atenção a aspectos relevantes da formação - como, por exemplo, a explicação dos professores.

A participação em atividades de extensão e pesquisa, bem como o curso de formação realizado (bacharelado ou licenciatura), mostrou-se associada ao nível de AE discente dos 
estudantes investigados no presente estudo. Conforme destacam Igue, Bariani e Milanesi (2008), o estudante necessita ser conscientizado da importância do engajamento precoce nas atividades oferecidas pela universidade, já que o desenvolvimento profissional não depende somente da influência exercida pela instituição sobre ele, mas, fundamentalmente, da intensidade de seu envolvimento com os recursos disponibilizados pelo ambiente acadêmico e com as diversas experiências externas de formação que são facilitadas no decorrer deste período.

As atividades de extensão podem ser compreendidas como um processo educativo, cultural e científico, sendo um elo imprescindível entre a universidade e os diversos setores da sociedade para que se consiga articular o ensino e a pesquisa de maneira indissociável (BIONDI; ALVES, 2011). A extensão é muito importante para a formação profissional, pois a aproximação do conhecimento acadêmico com a cultura popular cria um ambiente propício ao surgimento de novas aprendizagens e competências, que promove maior confiança e amadurecimento do estudante para lidar com as diversas situações vivenciadas no dia a dia de um profissional (NOZAKI; HUNGER; FERREIRA, 2011), além de oportunizar maior vínculo entre a teoria e a prática.

O Centro de Desportos (CDS/UFSC) oferece número considerável de projetos de extensão à comunidade (UNIVERSIDADE FEDERAL DE SANTA CATARINA, 2015), o que proporciona aos estudantes a oportunidade de realizar estágios em diferentes áreas de intervenção (tais como: dança; esportes coletivos e individuais; ginástica; natação; yoga) e atende aos interesses de grande parcela dos estudantes. Em investigação realizada com egressos e estudantes do antigo curso de licenciatura em Educação Física da UFSC, Mendes et al. (2006) constataram que a experiência profissional adquirida a partir do envolvimento com atividades de extensão e pesquisa elucidava a contribuição delas para a melhoria da qualidade da formação acadêmica e para o desenvolvimento satisfatório de competências acadêmicas e profissionais.

Um aspecto a destacar é que a maioria dos projetos existentes no CDS/UFSC oferece atividades mais direcionadas às especificidades do curso de bacharelado (como, por exemplo, grupos de reabilitação cardíaca, treinamento de equipes esportivas universitárias, iniciação e aperfeiçoamento em modalidades esportivas) (UNIVERSIDADE FEDERAL DE SANTA CATARINA, 2015). Mesmo que o estudante do curso de licenciatura esteja inserido em algum desses projetos, as dificuldades de atuação poderão ser maiores em comparação com aquelas enfrentadas pelo estudante do curso de bacharelado, que possui disciplinas obrigatórias como Atividade Física para Grupos Especiais, Condicionamento Físico e Treinamento Esportivo.

Os benefícios da participação em projetos de extensão para o crescimento profissional também são apontados por discentes investigados em outros estudos, dentre os quais se destacam: melhor percepção de segurança dos estudantes ao desenvolver diversos tipos de ações na prática diária (NOZAKI; HUNGER; FERREIRA, 2011); adoção de postura profissional mais humanizada e tomada de decisões reflexivas, autônomas e mais preocupadas com as necessidades da comunidade; desenvolvimento de qualidades como o espírito de equipe, a iniciativa e a criatividade (BIONDI; ALVES, 2011).

A atividade de pesquisa, exigência cada vez maior tanto do ambiente acadêmico como da própria sociedade, vem encontrando espaço crescente nas universidades brasileiras, especialmente após a consolidação da pós-graduação stricto sensu e o surgimento de laboratórios e de grupos de pesquisa nas mais diversas áreas do conhecimento (FREIRE; VERENGUER; REIS, 2002). A realização da pesquisa pelos discentes na formação inicial é 
fundamental para o desenvolvimento de atitudes investigativas e proativas, pois possibilita a intervenção crítica nas realidades acadêmica e profissional. Neste sentido, considerando que a observação e a problematização de situações são comuns à prática cotidiana, seu aprimoramento pode ser facilitado com a inserção do estudante em grupos e projetos de pesquisa desde as fases iniciais do curso (MENDES et al., 2006).

A oferta de atividades de extensão e de pesquisa, portanto, é capaz de potencializar o desenvolvimento de competências acadêmico-profissionais que podem contribuir, dentre outras formas, para a melhoria relacionada ao autoconceito, ao comportamento exploratório e ao envolvimento acadêmico dos estudantes, o que traz implicações diretas para a melhoria da $A E$ discente na formação inicial. Certamente, também são necessárias atitudes proativas por parte dos estudantes para que a inserção em grupos de pesquisa seja bem-sucedida. A atual proposta curricular oferece, na primeira fase de ambos os cursos, disciplinas que abordam conteúdos introdutórios da Educação Física - os quais procuram, dentre outros objetivos, esclarecer ao estudante acerca de projetos e de grupos de pesquisa existentes no CDS/UFSC. Cabe ao discente, desta maneira, reconhecer a temática investigativa de seu interesse e, a partir desta constatação, buscar informações junto aos pesquisadores e ambientes que mais se aproximem de seus interesses.

A AE discente se mostrou associada ao IAA e ao SDI dos estudantes investigados no presente estudo. No que diz respeito ao desempenho acadêmico (IAA), as evidências obtidas encontram grande respaldo na literatura científica internacional (BOUFFARD et al., 2005, BEMBENUTTY, 2007, ELIAS, 2008, AL KHATIB, 2010, CENDALES; VARGAS-TRUJILLO; BARBOSA, 2013), enquanto que, no âmbito nacional, ainda há carência de estudos que explorem a referida temática. No que diz respeito ao SDI, as evidências do presente estudo também encontram suporte na literatura consultada. Neste sentido, investigações sugerem que, quanto maior for o nível de $A E$, mais elevados serão, também, os níveis de esforço e de persistência mobilizados para se atingir a meta ou realização previamente estabelecida (BANDURA, 1977; 1997), lembrando que tais características são habitualmente encontradas em indivíduos mais motivados intrinsecamente (BOUFFARD et al., 2005, AL KHATIB, 2010).

Os estudantes com $\mathrm{AE}$ maior costumam regular melhor suas condições e estratégias de estudo (BEMBENUTTY, 2007), ajustando-se de maneira mais adequada às demandas acadêmico-pessoais e profissionais (CAZAN, 2012) e, quando necessário, buscando por auxílio de maneira mais frequente em comparação aos estudantes com baixo nível de AE (WILLIAMS; TAKAKU, 2011). Como consequência do maior ajustamento acadêmico, os estudantes com maior percepção de AE tendem a se sentir mais autoconfiantes (WANG; CASTAÑEDASOUND, 2008) e mais intrinsecamente motivados ou autodeterminados (AL KHATIB, 2010) para se esforçar em prol de determinada meta acadêmica que seja significativa para si próprio (BOUFFARD et al., 2005). Ao se considerar, também, que as metas dos estudantes com maior $A E$ costumam ser mais elevadas, bem como que envolvem a aprendizagem ou a maestria em determinado conteúdo ou situação (HSIEH; SULLIVAN; GUERRA, 2007), torna-se possível compreender as evidências encontradas no presente estudo a respeito da associação entre a AE discente, o SDI e o desempenho acadêmico (IAA).

Não foi encontrada associação entre o nível de progressão no curso e o nível de AE discente dos estudantes investigados. Em estudo realizado com 169 estudantes universitários ingressantes de diferentes cursos, Guerreiro-Casanova e Polydoro (2011) buscaram comparar 
a $A E$ de estudantes universitários entre o primeiro e o segundo período letivo. As evidências encontradas apontaram para uma diminuição do nível de $\mathrm{AE}$ no período pesquisado, o que pode demonstrar que, após as vivências acadêmicas dos estudantes no decorrer do período letivo, suas percepções se tornaram mais ajustadas ao contexto ambiental, bem como às capacidades individuais. Neste sentido, acredita-se que 0 achado do presente estudo pode estar relacionado ao ajustamento da percepção dos estudantes, ao longo da formação, em relação ao contexto universitário e às suas próprias capacidades. Além disso, devem ser consideradas as influências simultâneas de ordem pessoal, acadêmica, familiar e profissional, as quais podem contribuir para a construção das percepções de AE discente, mas que não foram objeto de estudo da presente investigação.

Embora possam ser feitas suposições que justifiquem a não existência de associação entre a $A E$ discente e o nível de progressão dos estudantes nos cursos, as evidências do presente estudo indicam a necessidade de refletir sobre a proposta organizacional dos cursos de formação oferecidos, na tentativa de que a forma de apresentação dos conteúdos contribua para torná-los mais significativos e impactantes aos estudantes. Ao investigarem os processos formativos utilizados no curso de bacharelado em Educação Física da instituição analisada no presente estudo, Milistetd et al. (2014) identificaram que as aulas teórico-práticas representavam as situações de aprendizagem utilizadas com maior frequência. Neste sentido, o excesso de aulas teóricas, a abstração de conteúdos em relação à prática e a superficialidade dos conteúdos abordados, em detrimento da promoção de vivências que oportunizassem a ocorrência de situações de aprendizagem experiencial, foram consideradas as principais fragilidades do curso investigado (MILISTETD et al., 2014). Ao reconhecer a importância da experiência direta e das experiências vicárias para o desenvolvimento de percepções positivas de AE (BANDURA, 1997), pode-se compreender com maior clareza que os cursos de formação inicial investigados no presente estudo ainda carecem de estratégias didático-pedagógicas que os tornem mais significativos e impactantes a seus estudantes.

\section{CONSIDERAÇÕES FINAIS}

A presente investigação possibilitou analisar as relações entre a $A E$ discente $e$ determinados aspectos da formação inicial realizada por estudantes universitários de Educação Física, com destaque para as associações das percepções dos estudantes investigados com determinadas características pessoais, acadêmicas e profissionais.

As evidências encontradas revelaram diferenças estatisticamente significativas no nível de $\mathrm{AE}$ discente quando consideradas as variáveis sexo, curso realizado, participação em atividades de pesquisa e extensão, Índice de Aproveitamento Acumulado e Índice de Autodeterminação, o que destaca a importância e a influência que variáveis pessoais e acadêmicas podem ter sobre as percepções individuais de AE dos estudantes investigados, e destas sobre o comportamento discente frente à formação.

A complexidade das relações estabelecidas entre os estudantes e sua formação, bem como a influência de inúmeros fatores pessoais e profissionais que extrapolam o espaço-tempo universitário, auxilia a encontrar possíveis explicações para o fato de não ter sido encontrada diferença no nível de AE dos estudantes investigados quando considerado o nível de progressão na formação inicial. No entanto, também se evidencia a necessidade de estimular a reflexão 
da comunidade acadêmica sobre a proposta organizacional vigente dos cursos na tentativa de superar as limitações decorrentes deste modelo atual que, ao priorizar excessivamente as situações de aprendizagem mediadas sob a forma de aulas expositivas, não parece conferir muito sentido e significado à aprendizagem discente. Neste sentido, é necessário promover maior valorização das experiências prévias que os estudantes já possuem, bem como ampliar a frequência de situações experienciais de aprendizagem, com o intuito de exercer maior impacto sobre os níveis de autoconfiança, de segurança e, consequentemente, de AE dos estudantes.

A continuidade do acompanhamento dos níveis de AE discente na formação inicial em Educação Física é sugerida, assim como a ampliação de estudos que busquem compreender com maior aprofundamento as complexas relações entre a $A E$, a motivação discente e a formação inicial universitária. Acredita-se que as investigações futuras poderiam avançar para além do delineamento transversal, por meio da utilização de abordagens retrospectivas e longitudinais que levassem em consideração as constantes mudanças de ordem pessoal (interna) e contextual (externa) que contribuem para a construção das percepções individuais de AE discente.

\section{REFERÊNCIAS}

AL KHATIB, Saleh Ahmed. Meta-cognitive self-regulated learning and motivational beliefs as predictors of college students' performance. International Journal for Research in Education, Washington, n. 27, p. 57-72, 2010.

BANDURA, Albert. A evolução da Teoria Social Cognitiva. In: BANDURA, Albert; AZZI, Roberta Gurgel; POLYDORO, Soely Aparecida Jorge. (Org.). Teoria social cognitiva: conceitos básicos. Porto Alegre: Artmed, 2008. p. 15-42.

BANDURA, Albert. Self-efficacy, the exercise of control. New York: Freeman, 1997.

BANDURA, Albert. Self-efficacy: toward a unifying theory of behavioral change. Psychological Review, Washington, v. 84, n. 2, p. 191-215, 1977.

BEMBENUTTY, Héfer. Self-regulation of learning and academic delay of gratification: Gender and ethnic differences among college students. Journal of Advanced Academics, Austin, v. 18, n. 4, p. 586-616, 2007.

BIONDI, Daniela; ALVES, Gabriela Cardozo. A extensão universitária na formação de estudantes do curso de Engenharia Florestal - UFPR. Revista Eletrônica do Mestrado em Educação Ambiental, Curitiba, v. 26, p. 209-224, 2011.

BOUFFARD, Thérèse et al. Influence of achievement goals and self-efficacy on students' selfregulation and performance. International Journal of Psychology, Paris, v. 40, n. 6, p. 373384, 2005.

BRASIL. Conselho Nacional de Educação (CNE). Resolução n. 1, de 18 de fevereiro de 2002a. Institui Diretrizes Curriculares Nacionais para a Formação de Professores da Educação Básica, em nível superior, curso de licenciatura, de graduação plena. Diário Oficial da União, Brasília, 4 mar. 2002a. Seção 1, p. 8. 
BRASIL. Resolução n. 2, de 19 de fevereiro de 2002b. Institui a duração e a carga horária dos cursos de Licenciatura, de Graduação Plena, de Formação de Professores da Educação Básica em nível superior. Diário Oficial da União, Brasília, 4 mar. 2002b. Seção 1, p. 9.

BRASIL. Resolução n. 7, de 31 de março de 2004. Institui as Diretrizes Curriculares Nacionais para os cursos de graduação em Educação Física, em nível superior de graduação plena.

Diário Oficial da União, Brasília, 5 abr. 2004. Seção 1, p. 18.

CAZAN, Ana-Maria. Self-regulated learning strategies - predictors of academic adjustment. Procedia: Social and Behavioral Sciences, Amsterdam, v. 33, p. 104-108, 2012.

CELIK, Kazim. The relationship between individual innovativeness and self-efficacy levels of student teachers. International Journal of Scientific Research in Education, Port Harcourt, v. 6, n. 1, p. 56-67, 2013.

CENDALES, Boris; VARGAS-TRUJILLO, Elvia; BARBOSA, Camila. Factores psicológicos asociados al desempeño académico en los cursos universitarios de estadística: diferencias por sexo y área de titulación. Avances en Psicología Latinoamericana, Bogotá, v. 31, n. 2, p. 363375, 2013.

ELIAS, Rafik Z.; Anti-intellectual attitudes and academic self-efficacy among Business students. Journal of Education for Business, Washington, v. 84, n. 2, p. 110-116, 2008.

ELIAS, Steven M.; LOOMIS, Ross J. Utilizing need for cognition and perceived self-efficacy to predict academic performance. Journal of Applied Social Psychology, Washington, v. 32, n. 8, p.1687-1702, 2002.

FOLTZ, Barbara McDonald; LUZZO, Darrell Anthony. Increasing the career decision-making selfefficacy on nontraditional college students. Journal of College Counseling, Alexandria, v. 1, p. 35-44, 1998.

FREIRE, Elisabete dos Santos; VERENGUER, Rita de Cássia Garcia; REIS, Marise Cisneiros da Costa. Educação Física: pensando a profissão e a preparação profissional. Revista Mackenzie de Educação Física e Esporte, São Paulo, v. 1, n. 1, p. 39-46, 2002.

GUAY, Frédéric; MAGEAU, Geneviève; VALLERAND, Robert. On the hierarchical structure of self-determined motivation: A test of top-down, bottom-up, reciprocal, and horizontal effects. Personality and Social Psychology Bulletin, Washington, v. 29, n. 8, p. 992-1004, 2003.

GUERREIRO-CASANOVA, Daniela Couto; POLYDORO, Soely Aparecida Jorge. Autoeficácia na formação superior: percepções durante o primeiro ano de graduação. Psicologia: Ciência e Profissão, Brasília, v. 31, n. 1, p. 50-65, 2011.

HSIEH, Pei-Hsuan; SULLIVAN, Jeremy R.; GUERRA, Norma R. A closer look at college students: Self-efficacy and goal orientation. Journal of Advanced Academics, Austin, v. 18, n. 3, p. 454-476, 2007.

IGUE, Érica Aparecida; BARIANI, Isabel Cristina Dib; MILANESI, Pedro Vitor Barnabé. Vivência acadêmica e expectativas de universitários ingressantes e concluintes. Psico-USF, Bragança Paulista, v. 13, n. 2, p. 155-164, 2008.

KOMARRAJU, Meera; NADLER, Dustin. Self-efficacy and academic achievement: Why do implicit beliefs, goals, and effort regulation matter? Learning and Individual Differences, Greenwich, v. 25, p. 67-72, 2013. 
LI, Ming-Hui; NISHIKAWA, Takeshi. The relationship between active coping and trait resilience across U.S. and Taiwanese college student samples. Journal of College Counseling, Alexandria, v. 15, p. 157-171, 2012.

LOPES, Ana Rita; TEIXEIRA, Maria Odília. Projetos de carreira, autoeficácia e sucesso escolar em ambiente multicultural. Revista Brasileira de Orientação Profissional, Ribeirão Preto, v. 13, n. 1, p. 7-14, jan./jun., 2012.

MARÔCO, João. Análise estatística com o SPSS Statistics. 5. ed. Pacific Grove: Brooks/Cole, 2010.

MASCARENHAS, Suely Aparecida do Nascimento. Avaliação da autoeficácia acadêmica de estudantes da UFAM/Amazônia. Revista Amazônica, Humaitá, v. 4, n. 1, p. 67-79, 2010.

MENDES, Evandra Hein et al. Avaliação da formação inicial em Educação Física: um estudo Delphi. Revista da Educação Física/UEM, Maringá, v. 17, n. 1, p. 53-64, 2006.

MILISTETD, Michel et al. Coaching and Coach Education in Brazil. International Sport Coaching Journal, Champaign/lL, v. 1, p. 165-172, 2014.

NOZAKI, Joice Mayumi; HUNGER, Dagmar; FERREIRA, Lílian Aparecida. Reflexões sobre um projeto de extensão universitária na formação/atuação do docente de Educação Física. In: CONGRESSO BRASILEIRO DE CIÊNCIAS DO ESPORTE, 17, CONGRESSO INTERNACIONAL DE CIÊNCIAS DO ESPORTE, 4., 2011, Porto Alegre. Anais... Porto Alegre, 2011. Disponível em: <http://cbce.tempsite.ws/congressos/index.php/conbrace2011/2011>. Acesso em: 21 nov. 2014.

OZAN, Ceyhun et al. A study on the university self-regulated learning strategies skills and selfefficacy perceptions in terms of different variables. Procedia: Social and Behavioral Sciences, Amsterdam, v. 46, p. 1806-1811, 2012.

PENG, Cuixin. Self-regulated learning behavior of college students of Science and their academic achievement. Physics Procedia, Amsterdam, v. 33, p. 1446-1450, 2012.

POLYDORO, Soely Aparecida Jorge; GUERREIRO-CASANOVA, Daniela Couto. Escala de Autoeficácia na Formação Superior: Construção e estudo de validação. Avaliação Psicológica, Porto Alegre, v. 9, n. 2, p. 267-278, 2010.

SÁNCHEZ ROSAS, Javier. Búsqueda de ayuda académica, autoeficacia social académica y emociones de logro en clase en estudiantes universitarios. Revista Argentina de Ciencias del Comportamiento, Córdoba, v. 5, n. 1, p. 35-41, 2013.

SANTOS, Maria José Etelvina dos. O Professor ecológico no contexto da instituição escolar. Revista FACED, Salvador, n. 15, p. 111-125, 2009.

SAWTELLE, Vashti; BREWE, Eric; KRAMER, Laird H. Exploring the relationship between selfefficacy and retention in introductory Physics. Journal of Research in Science Teaching, Reston, v. 49, n. 9, p. 1096-1121, 2012.

SILVA, Afonsa Janaína da; IAOCHITE, Roberto Tadeu; AZZI, Roberta Gurgel. Crenças de autoeficácia de licenciandos em Educação Física. Motriz, Rio Claro, v. 16, n. 4, p. 942-949, 2010.

SOBRAL, Dejano. Motivação do aprendiz de Medicina: Uso da Escala de Motivação Acadêmica. 
SOBRAL, Dejano. Autodeterminação da motivação em alunos de Medicina: Relações com motivos de escolha da opção e intenção de adesão ao curso. Revista Brasileira de Educação Médica, Rio de Janeiro, v. 32, n. 1, p. 56-65, 2008.

THOMAS, Jerry R.; NELSON, Jack K.; SILVERMAN, Stephen J. Métodos de Pesquisa em Atividade Física. Porto Alegre: Artmed, 2007.

UNIVERSIDADE FEDERAL DE SANTA CATARINA. Resolução no 17/CUn/97, de 30 de setembro de 1997. Dispõe sobre o Regulamento dos Cursos de Graduação da UFSC. Florianópolis, 1997. Disponível em: <http://antiga.ufsc.br/paginas/downloads/UFSC Resolucao N17 CUn97.pdf acesso>. Acesso em: 16 jul. 2015.

UNIVERSIDADE FEDERAL DE SANTA CATARINA. Extensão: atividades física para comunidade. Disponível em: <http://portalcds.ufsc.br/extensao-atividades-fisicas-para-acomunidade/>. Acesso em: 10 fev. 2015.

VALLERAND, Robert et al. On the assessment of intrinsic, extrinsic, and amotivation in education: Evidence on the concurrent and construct validity of the Academic Motivation Scale. Educational and Psychological Measurement, Washington, v. 53, p. 159-172, 1993.

VEGA, Humberto Blanco et al. Autoeficacia percibida en conductas académicas: Diferencias entre hombres y mujeres. Revista Mexicana de Investigación Educativa, México, v. 17, n. 53, p. 557-571, 2012.

VELIZ-BURGOS, Alex; URQUIJO, Pedro Apodaca. Niveles de auto concepto, autoeficacia académica y bienestar psicológico en estudiantes universitarios de la Ciudad de Temuco. Salud e Sociedad, Antofagasta, v. 3, n. 2, p. 131-150, 2012.

VIEIRA, Diana; COIMBRA, Joaquim Luís. A autoeficácia na transição para o trabalho. In: AZZI, Roberta Gurgel; POLYDORO, Soely Aparecida Jorge (Org.). Autoeficácia em diferentes contextos. Campinas: Alínea, 2006. p. 25-58.

WANG, Chia-Chih D.; CASTAÑEDA-SOUND, Carrie. The role of generational status, selfesteem, academic self-efficacy, and perceived social support in college students' psychological well-being. Journal of College Counseling, Alexandria, v. 11, p. 101-118, 2008.

WILLIAMS, James D; TAKAKU, Seiji. Help seeking, self-efficacy, and writing performance among college students. Journal of Writing Research, Antuerpia, v. 3, n. 1, p. 1-18, 2011.

Apoio: Coordenadoria de Aperfeiçoamento de Pessoal de Nível Superior (CAPES); Conselho Nacional de Desenvolvimento Científico e Tecnológico (CNPq). 